Abstracts of papers presented at the seventh Mammalian Genetics and Development Workshop held at the Wellcome Trust Building, Euston Road, London on 20-22 November 1996

Edited by: DENNIS STEPHENSON

The Galton Laboratory, University College London, Wolfson House, 4 Stephenson Way, London, UK

Towards the functional characterization of the mouse Xist gene promoter

S. A. SHEARDOWN, A. NEWALL, D. P. NORRIS, C. JOHNSTON, S. RASTAN, and N. BROCKDORFF

Comparative Biology Group, MRC Clinical Sciences Centre, Royal Postgraduate Medical School, Hammersmith Hospital, Du Cane Road, London W12 ONN, UK

The Xist gene is a candidate for the $\mathrm{X}$ inactivation centre and we have recently shown that Xist transcription is necessary for $\mathrm{X}$ inactivation in the mouse. Xist transcription is repressed in $\mathrm{XX}$ diploid embryonic stem cells and both $\mathrm{X}$ chromosomes are active. Upon differentiation one Xist allele becomes expressed, preceding cis inactivation of that $\mathrm{X}$ chromosome, while the Xist allele on the active $\mathrm{X}$ chromosome remains silent. This is normally a random process, either allele having an equal probability of being activated. To identify sequence elements involved with Xist regulation we have surveyed $10 \mathrm{~kb}$ of the Xist promoter for DNaseI hypersensitive sites and have assayed their properties using a reporter gene system. Functional elements identified thus far affect reporter gene expression in a complex manner but are not sufficient to permit appropriate expression. A detailed examination of the Xist minimal promoter has revealed several previously uncharacterized transcription factor binding sites. One of these, designated $\mathrm{XPF} 1$, is a potential regulatory switch since it overlaps a canonical CAAT box and an SP1 site, and can displace these factors in vitro. Some implications of these data regarding the regulation of random monoallelic Xist expression will be discussed.
Regulation of the Xist minimal promoter studied by microinjection in preimplantation embryos and in transgenic mice

TETSUYA GOTO, ELISABETH CHRISTIANS and MARILYN MONK

Molecular Embryology Unit, Institute of Child Health, 30 Guilford Street, London WC1N 1EH, UK

The mouse Xist gene is expressed only from an inactive $\mathrm{X}$ chromosome and plays a role in $\mathrm{X}$ chromosome inactivation in female development and in male spermatogenesis. Differential expression of the paternal Xist in preimplantation development has also been implicated in imprinted paternal X-inactivation in extra-embryonic tissues. To identify important regulatory elements in the Xist promoter, we have made a construct ligating a $233 \mathrm{bp}$ PCRamplified Xist promoter fragment $(-220$ to +13$)$ to the firefly luciferase reporter gene and the SV40 enhancer. Following injection of the construct into 1cell embryos, luciferase activity is detected at the 2-cell stage. Methylation of the construct by site-specific methylases before injection results in a decrease in luciferase activity. We have also created transgenic lines harbouring the $2.5 \mathrm{~kb}$ linearized Xist-luciferase insert. In all six lines established, luciferase is highly expressed in the testis. In contrast, only background luciferase activity is observed in the liver, kidney, muscle, brain and ovary. A DNA methylation study shows that the transgene is undermethylated in the testis compared with other tissues examined. The regulatory sequences involved in promoter activation, imprinting and tissue-specificity are being investigated.

\section{Determination of the active chromatin domain of the expressed Xist allele in mouse}

VERONICA MCCABE, STEVE SHEARDOWN and NEIL BROCKDORFF

Section of Comparative Biology, CSC Hammersmith Hospital, Du Cane Road, London, W12 0NN

$\mathrm{X}$ chromosome inactivation is the genetic silencing of one of the two $\mathrm{X}$ chromosomes in female mammals. The exact mechanism is still not completely understood but it is now known that the Xist gene, a 
candidate for the $\mathrm{X}$ inactivation centre, is required. The inactive $\mathrm{X}$ is heterochromatic and transcriptionally silent, so it is of interest to determine the extent of the active chromatin domain around Xist and identify what features distinguish the Xist domain from the surrounding inactive chromatin. We have examined the coding region and $60 \mathrm{~kb}$ of the noncoding $5^{\prime}$ region of Xist by looking at two frequently associated features of transcriptionally active chromatin: DNaseI sensitivity and histone $\mathrm{H} 4$ acetylation. Immunoprecipitation of chromatin fragments with antibodies specific for $\mathrm{H} 4$ acetylated at specific lysine residues and the subsequent analysis of the genic and $5^{\prime}$ region has identified both acetylated and nonacetylated regions. Within the centre of Xist we see higher levels of acetylation compared with the $5^{\prime}$ flanking regions. Interestingly we see similar results in males where Xist is not expressed. The expressed and silent Xist alleles have also been examined at the level of DNaseI sensitivity using a PCR-based assay. This has demonstrated that within the genic region the expressed Xist allele is more sensitive to DNaseI. Regions 20 and $50 \mathrm{~kb} \mathrm{5}$ of the start site do not display this feature. Thus these data suggest that the proximal limit of the Xist domain lies within $20 \mathrm{~kb}$ of the start site.

\section{Histone acetylation in mouse embryonic stem cells}

LAURA O'NEILL, ANN M. KEOHANE and
BRYAN TURNER
Chromatin and Gene Expression Group, Department of
Anatomy, University of Birmingham, Birmingham, UK

We have previously observed a difference between the level of acetylated $\mathrm{H} 4$ within euchromatin and heterochromatin and a transient change in the acetylation status of heterochromatin as cells begin to differentiate (EMBO J. 14, 1995). Following on from these studies, we have begun to investigate the changes that occur in the acetylation status of $\mathrm{H} 4$ and gene activity as embryonic stem cells are induced to differentiate. Removal of leukaemia inhibitory factor from the medium causes these cells to differentiate into embryoid bodies. Using immunofluorescence we have followed the changes in the acetylation status of the $\mathrm{X}$ chromosome. In undifferentiated female embryonic stem cells there are two active $\mathrm{X}$ chromosomes, both of which label with antibodies to acetylated $\mathrm{H} 4$. As the cells differentiate one of the $\mathrm{X}$ chromosomes becomes deacetylated (i.e. weakly stained) from day 4 onwards. Xist expression, down regulation of $\mathrm{X}$ linked genes and late replication all occur earlier (days 1-4). In parallel experiments we have used immunoprecipitation with antibodies to acetylated $\mathrm{H} 4$ to show that changes in the acetylation status of both X-linked and autosomal genes occur as these cells differentiate. Furthermore, DNA from heterochromatic regions shows a moderate level of acetylation in undifferentiated cells, but by day 7 is associated with nonacetylated $\mathrm{H} 4$, as in adult cells - findings that are entirely consistent with the observations by immunofluorescence.

Fertile $\mathrm{X}^{\mathrm{Y}^{\star}} \mathrm{O}$ male mice: evidence for a mutation which circumvents the 'meiotic quality control'

TRISTAN RODRIGUEZ, TERESA ODORISIO and PAUL S. BURGOYNE

Laboratory of Developmental Genetics, National Institute for Medical Research, The Ridgeway, Mill Hill, London NW7 1AA, UK

We have previously reported that $\mathrm{X}^{\mathrm{Y}} \mathrm{O}$ male mice, which have an $\mathrm{X}$ and a $\mathrm{Y}$ chromosome attached by a shared pseudoautosomal region, have an almost total meiotic block at the first meiotic metaphase. The arrested metaphase I spermatocytes are eliminated via a p53-independent pathway. This spermatocyte arrest and elimination has been interpreted as a manifestation of a 'meiotic quality control' which acts to remove meiotic cells which have not achieved full chromosomal synapsis. With respect to the $\mathrm{X}-\mathrm{Y}$ bivalent, pseudoautosomal synapsis is thought to be sufficient to avoid triggering this 'meiotic quality control'; in $\mathrm{X}^{\mathrm{Y}} \mathrm{O}$ males pseudoautosomal synapsis is prevented because there is a single pseudoautosomal region. Recently we identified a fertile $\mathrm{X}^{\mathrm{Y} *} \mathrm{O}$ male which proved to have over 1 million sperm per caput epididymis. Subsequent pedigree analysis has indicated that fertility is primarily due to a single major autosomal factor which allows the production of several hundred thousand sperm. A second minor autosomal factor, which of itself rarely if ever allows fertility, is necessary to achieve sperm counts of over 1 million. Preliminary data suggest that the major factor may be lethal when homozygous. We are currently introducing the mutation into other mice with chromosomally induced sterility.

Identification of $H-Y$ epitopes in $S m c y$ and other genes mapping to the mouse $Y$ chromosome short arm

D. SCOTT, ${ }^{1}$ I. EHRMANN,${ }^{1}$ P. ELLIS, ${ }^{1}$ M. MITCHELL ${ }^{2}$ A. GREENFIELD ${ }^{3,4}$, P. KOOPMAN $^{3}$ and E. SIMPSON ${ }^{1}$

${ }^{1}$ Clinical Sciences Centre, RPMS, London, UK. ${ }^{2}$ Inserm Unite 406, Marseille, France. ${ }^{3}$ Centre for Molecular and Cellular Biology, Brisbane, Australia. ${ }^{4} M R C$ Mammalian Genetics Unit, Harwell, UK.

Two $H-Y$ epitopes - mouse $H-Y K^{k}$ and human $H$ $Y B 7$ - were recently identified as products of $S m c y$, a gene mapping to the short arm of the mouse $\mathrm{Y}$ 
chromosome. The $H-Y K^{k}$ epitope was defined as the peptide, TENSGKDI. This differs by five amino acids from the $\mathrm{X}$ chromosome homologue, which does not stimulate an $H-Y$ specific $\mathrm{T}$ cell clone. Deletion mutants not expressing this peptide fail to stimulate the $\mathrm{T}$ clone. A $5 \mathrm{~kb}$ cDNA expressing most of the Smcy sequence was synthesized by PCR from a $5^{\prime}$ Smcy cDNA clone. Following transfection, this gave rise to $H-Y K^{k}$ expression, as did a $3^{\prime}$ cDNA clone which also encoded the peptide. Smcy was also found to express an $H-Y D^{k}$ epitope, which has been localized to two exons mapping within a $9 \mathrm{~kb}$ genomic fragment (MEM $14 \mathrm{~B}$ ) that lies $5^{\prime}$ of that expressing the $\mathrm{H}-Y K^{k}$. Smcy does not appear to encode other $H-Y$ epitopes, suggesting that there are other genes in this region from which the other $H-Y$ epitopes are derived. Another ubiquitously expressed $\mathrm{Y}$ chromosome gene, Uty, also mapping to this region, was found to express an $H-Y D^{b}$ epitope. The $H-Y D^{b}$ peptide is encoded in the $5^{\prime}$ region of the Uty cDNA. It differs from that encoded by Utx, the $\mathrm{X}$ chromosome homologue of this gene, by three amino acids. The $\mathrm{N}$ residue at position 5 is the anchor residue and is required for binding to $H-2 D^{b}$, while $\mathrm{M}$ at position 6 and $\mathrm{D}$ at 7 are critical for $\mathrm{T}$ cell recognition.

Identification of a gene on the mouse $Y$ chromosome which is homologous to a translation factor and may encode an $H$ - $Y$ antigen

INGRID EHRMANN ${ }^{1}$, DIANE SCOTT $^{1}$, MICHAEL MITCHELL ${ }^{2}$, SOPHIE MAZEYRAT ${ }^{2}$, MARK GAVIN ${ }^{3}$, ELIZABETH SIMPSON ${ }^{1}$ and PAM ELLIS ${ }^{1}$

${ }^{1}$ Clinical Sciences Centre, RPMS, London, UK; ${ }^{2}$ Inserm Unité 406, Marseille, France; ${ }^{3}$ University of Washington, Seattle, USA

$S x r^{b}$ mice which have a deletion of the mouse Y chromosome are known to have spermatogenic defects. It has also been shown that the $H-Y$ antigens (minor transplantation antigens involved in graft rejection) map to this interval. We are interested in cloning genes from the $S x r^{b}$ interval in order to study $H-Y$ antigens and spermatogenesis. Recently, we cloned a gene by differential display which maps to the $S x r^{b}$ interval and is homologous to a human translation factor. We have also found that two non-Y copies of $T Y$ (translation factor on the $\mathrm{Y}$ ) exist in the mouse. The Y gene is expressed in all tissues tested by Northern blot analysis, suggesting that it could be an $H-Y$ antigen. $H-Y$ antigens are known to be ubiquitously expressed. We have identified two $H$ - $Y$ antigens $-H-Y K^{k}$ and $H-Y D^{b}$ - and the genes that they are derived from already. We have also located $H-Y D^{k}$ activity in the $S m c y$ gene. Therefore, $T Y$ may encode either $H-Y A^{b}$ or $H-Y E^{k}$. We are in the process of transfecting cell lines expressing the appropriate class II MHC molecule with the $T Y$ gene and assaying for $H-Y$ activity. Regardless of whether $T Y$ encodes an $H-Y$ antigen, the existence of a translation factor on the $Y$ chromosome suggests that it may be involved in translating male-specific proteins.

Immuno- and molecular cytogenetic investigations of meiotic recombination and interference in mouse and human spermatocytes

\section{A. HULTÉN and A. BARLOW \\ LSF Research Unit, Regional Genetic Services, Birmingham Heartlands Hospital, Yardley Green Road, Birmingham B9 5PX, UK}

The mechanisms of mammalian meiotic recombination and genetic interference are largely unknown. It seems clear, though, that the two chromosomes within each pair are aligned at meiosis I with synapsis of homologous sequences starting at termini in the formation of the so called synaptonemal complex (SC). In order to obtain base-line information on the chromatin organization of the $\mathrm{SC}$ we have used a combination of immuno- and molecular cytogenetics to study the distribution of both repetitive and single copy DNA sequences relative to the SC. Telomeric sequences uniquely remain strongly associated with the SC, whilst chromosome pairs, centromeric and heterochromatic sequences hybridize to chromatin loops that associate with the SC only at their bases. In an attempt to elucidate both the mechanisms and distribution of recombination events we have also used antibodies against the recombinogenic proteins Rad51 (a recA homologue) and MLH1 (a mis-match repair enzyme) in the same way. Rad51, which is believed to be involved in DNA homology search and strand invasion, is localized as SC-associated foci in meiosis I prophase spermatocytes from zygotene to late pachytene. Zygotene cells contain numerous foci of anti-Rad51 labelling, with the brightest found in synapsing/synapsed regions. Pachytene cells show a different distribution, with synapsed regions either unlabelled or lightly labelled, whilst the XY and any unsynapsed autosomal segments become heavily labelled. We conclude that, contrary to previous suggestions (Plug et al., PNAS 1996, 12, 5920-4), Rad51 is not involved in long-distance chromosome alignment, but instead functions in the DNA homology search at synapsis. The anti-MLH1 antibody demonstrates bright foci that co-localise with SCs from mid- to late pachytene. Their frequency distribution appears to coincide with that of chiasmata, thus providing a new tool both for evaluation of interference distances and for precise mapping of crossing-over events in relation to strategic chromosome markers. 
Localization of the intragenic recombination hotspot in the human phosphoglucomutase-1 (PGM1) locus

\section{S. P. YIP, Y. H. EDWARDS, D. A. HOPKINSON and D. B. WHITEHOUSE \\ MRC Human Biochemical Genetics Unit, Galton Laboratory, University College London, Wolfson House, 4 Stephenson Way, London NW1 2HE, UK}

The human PGM1 protein is highly polymorphic with four common alleles: $1+, 1-, 2+$ and $2-$. Current findings indicate that these isozyme alleles are generated by point mutations at two sites and the action of an intragenic recombination hotspot between them. The two sites are $18 \mathrm{~kb}$ apart in the gene, one in exon 4 leading to the $2 / 1$ polymorphism and the other in exon 8 accounting for the $+/-$ polymorphism. Here we report nucleotide polymorphisms in the introns of the gene that are being used to pinpoint the recombination hotspot. PCR-amplified fragments were analysed with single strand conformation polymorphism (SSCP) to look for polymorphisms. Four new markers have been found and characterized: one in intron 4, two in intron 6 and one in intron 7. For each marker, samples from three different populations (Caucasian, Chinese and Vietnamese) were typed, and its molecular basis was determined. Pair-wise allelic association analyses were performed to identify linkage disequilibrium between each marker and the $2 / 1$ or the $+/-$ site. With this population-based approach, we have been able to narrow down the region of recombination from the original $18 \mathrm{~kb}$ to a $2 \mathrm{~kb}$ segment bound by two markers flanking exon 7 .

How to make your primers last $\mathbf{1 0 0 0 0}$ times as many reactions, cost twice as much and (fairly) reliably detect single base differences in multiplex PCR (and related topics)

MICHAEL J. HOBART

MIP Unit, MRC Centre, Hills Road, Cambridge CB2 $2 Q H, U K$

The use of TAGed primers was introduced by Prof. Sir Alec Jeffreys for his investigations of VNTRs. The TAG sequence is an arbitrary 20 bases at the $5^{\prime}$ end of the primer, and it was designed for nested multiplex PCR without the products shortening. The PCR reaction is functionally separated into the templatespecific event and the amplification of the products thus produced. Different conditions can be used for each phase and the initial primer concentration can be very low (certainly $10^{-5}$ of normal). Under low primer conditions, allele-specific priming can be achieved over a wide range of conditions (temperatures). Thus it is possible to design primers to a common Tm and multiplex them in a single reaction. Both strands can be investigated at once, though this does mean that the primers overlap. In addition, the TAG can be used to 'bolt on' a target sequence for a TaqMan assay.

\section{How important are repetitive regions within develop- mentally relevant genes?}

JOHN M. HANCOCK

MRC Clinical Sciences Centre, Royal Postgraduate Medical School Hammersmith Hospital, Du Cane Road, London W12 ONN, UK

The coding regions of many developmentally relevant genes contain repetitive (simple) sequences prone to replication slippage. Experimental dissection of these regions has not provided strong evidence that they play an important role in the function of their gene products, although in Drosophila the slippagegenerated changes in hunchback are associated with some differences in expression pattern. However, analysis of simple sequences found in some developmental genes suggests that selection has acted on them, suggesting a potential role in morphological evolution.

CpG islands of chicken are concentrated on microchromosomes

HEATHER A. MCQUEEN, GIORGIA SIRIACO and ADRIAN P. BIRD

Institute of Cell and Molecular Biology, University of Edinburgh, Darwin Building, King's Buildings, Edinburgh EH9 3JR, UK

We are engaged in the study of $\mathrm{CpG}$ islands and their organization in the chicken genome. $\mathrm{CpG}$ islands are short unmethylated $\mathrm{CpG}$-rich sequences situated to the $5^{\prime}$ end of many vertebrate genes and are particularly GC-rich in the chicken. The chicken karyotype comprises 39 chromosome pairs of which at least 29 are microchromosomes. Microchromosomes account for only about $25 \%$ of the genomic DNA and are cytologically indistinguishable. Due to technical limitations microchromosomal genes are poorly represented in current genome maps. However, we have used a $\mathrm{CpG}$ island library, prepared from chicken, to demonstrate that $\mathrm{CpG}$ islands are concentrated on the microchromosomes. Our results imply a higher gene density for chicken microchromosomes than is seen in mammalian species. We are currently testing this hypothesis using cloned segments of microchromosomal chicken DNA. 


\section{Folate and protecting the fetus from environmental toxins}

VALERIE SMELT ${ }^{1}$, JASON POPE ${ }^{1}$, HELEN MARDON $^{2}$, CHRIS REDMAN ${ }^{2}$ and EDITH SIM ${ }^{1}$ ${ }^{1}$ Department of Pharmacology, Oxford University, Mansfield Road, Oxford OX1 3QT; ${ }^{2}$ Department of Obstetrics and Gynaecology, John Radcliffe Hospital, Headington, Oxford OX3 9DU, UK

An unborn baby encounters virtually all the chemicals in its mother's blood. Exposure is via the placenta, whose metabolic capabilities determine the chemicals reaching the baby [1]. Arylamine $N$-acetyltransferase type 1 (NAT1*) is involved in the metabolism of environmental toxins [2] and is active in the placenta at term [1]. Folate is essential for embryonic development [3]. Maternal excretion of one of its metabolites, para-acetamidobenzoyl-L-glutamate (apBGlu), increases dramatically during the second trimester of pregnancy [4]. NAT1* is postulated to be responsible for apBGlu production [5], hence could increased NAT1* activity during pregnancy be responsible for increased metabolite excretion? In turn, does this result in fetal protection from environmental toxins? The NAT1* activity of 32 different placentae and cord blood from seven individuals has been determined using para-aminobenzoic acid (p-aba) as substrate. Samples were collected from term Caesarean or vaginal deliveries. NAT1*-specific enzymic activity varied seven-fold amongst individuals. The NAT1* activity of cord blood was in the expected adult range and the variation in neonates matches the previously observed variation in the adult population [6]. Using antibody raised against expressed NAT1*, NAT1* has been detected quantitatively in placenta by Western blotting. Immunohistochemical staining confirms the cytoplasmic location of NAT $1 *$ in cells on the maternal and fetal faces of the placenta. [7].

1. Derewlany, L.O., Knie, B. \& Koren, G. (1994). Arylamine $N$-acetyltransferase activity of the human placenta. J. Pharmacol. Exp. Ther. 269, 756-60.

2. Deguchi, T. (1992). Physiology and molecular biology of arylamine $N$-acetyltransferases. Biomed. Res. 13, 231-42.

3. Scott, J.M., McPartlin, J. et al. (1993). Folate metabolism in pregnancy. Adv. Exp. Med. Biol. 338, 727-32.

4. McPartlin, J., Halligan, A., Scott, J.M., Darling, M. \& Weir, D.G. (1993). Accelerated folate breakdown in pregnancy. Lancet 341, 148-9.

5. Ward, A., Summers, M.J. \& Sim, E. (1995). Purification of recombinant human $N$-acetyltransferase type 1 (NAT1) expressed in E. coli and characterization of its potential role in folate metabolism. Biochem. Pharmacol. 49, 1759-67.

6. Ward, A., Hickman, D., Gordon, J.W. \& Sim, E. (1992). Arylamine $N$-acetyltransferase in human red blood cells. Biochem. Pharmacol. 44, 1099-104.
7. Pacifici, G.M., Bencini, C., Rane, A. (1986). Acetyltransferases in humans: development and tissue distribution. Pharmacology 32, 283-91.

\section{Three-dimensional studies of human craniofacial growth}

ROBIN HENNESSY, PAUL O'HIGGINS and SAM COBB

The Galton Laboratory and Departments of Medical Physics and Bio-Engineering and of Anatomy and Developmental Biology, University College London, London, UK

Traditional shape analysis or morphometrics is associated with the multivariate analysis of sets of inter-landmark distance measurements generally obtained with simple calipers. Recent advances in the study of biological shape have now brought together the powerful analytical machinery known as 'geometrical morphometrics' with advanced digitization technologies. The methods of geometric morphometrics preserve information about three-dimensional morphology at every stage of the statistical analysis and so allow statistical findings to be presented graphically and more readily interpreted. This paper will review the advances in these two fields and present preliminary results of a $3 \mathrm{D}$ study of human craniofacial growth. Fifteen skulls have been studied which span the age range from 24 weeks from conception to 14 years. The 3D coordinates of important facial landmarks have been extracted from the data and a statistical analysis of the landmark coordinates has been carried out to determine the principal characteristics of facial growth vector. This work, therefore, is an application of geometric morphometrics in the study of allometry. The methods can be readily extended to studies of comparative growth and development and so have enormous potential in evolutionary and developmental studies.

\section{Selection-induced gene expression in thymocytes}

DANIEL GRAF, AMANDA G. FISHER and MATTHIAS MERKENSCHLAGER

Lymphocyte Development Group, MRC Clinical Sciences Centre, Hammersmith Hospital, Du Cane Road, London W12 ONN, UK

$\mathrm{CD}^{+}$and $\mathrm{CD} 8^{+} \mathrm{T}$-cell lineages arise in the thymus from a common precursor. They develop along a shared pathway up to the $\mathrm{CD} 4^{+} \mathrm{CD} 8^{+}$double-positive stage. Then they undergo positive and negative selection and mature into single-positive $\mathrm{T}$ cells. During this maturation the thymocytes commit themselves to either the CD4 or CD8 T cell lineage. Thymocyte maturation to the single positive stage requires the interaction of TCR with MHC molecules 
expressed on thymic stroma. However, the molecular basis promoting this maturation is not well understood. To learn about this we exposed thymocytes from MHC-deficient mice to MHC-expressing thymic stroma cells in vitro. A modified differential display approach was used to identify genes induced after interaction of TCR with either MHC class I, MHC class II or both MHC class I and II. Among the genes isolated is Nur77, a member of the orphan steroid receptor superfamily, which has been implicated in negative selection. The identification and characterization of other genes induced early after TCR/MHC interaction should help to elucidate events associated with positive/negative selection and/or lineage commitment.

The caudal type homeobox protein CDX2 binds to the colon promoter of the carbonic anhydrase 1 gene

FELICITY DRUMMOND, JANE SOWDEN, KATIE MORRISON and YVONNE H. EDWARDS MRC Human Biochemical Genetics Unit, University College London, Wolfson House, 4 Stephenson Way, London NW1 $2 H E, U K$

Carbonic anhydrase 1 (CA1) is an abundant enzyme in colon epithelia. In the gastrointestinal tract carbonic anhydrase is vital for $\mathrm{NaCl}$ resorption, alkalinization of gut contents and absorption of short chain fatty acids. The CA1 gene has two promoters, one of which is specifically active in colon epithelia and the other in erythroid cells. We are investigating the factors which regulate CA1 expression from the colon-specific promoter. Using electrophoretic mobility shift assays to search a $650 \mathrm{bp}$ region which contains a colonspecific DNaseI hypersensitive site we have identified two copies of a motif which binds a colon specific factor. Competition assays and antibody studies indicate that this factor is identical to the homeodomain protein CDX2. Experiments in which CDX2 in an expression vector has been co-transfected with various CA1 promoter/reporter gene constructs into HeLa cells, have shown that CDX2 protein binds to the CA1 sequences and acts to upregulate a minimal promoter. Furthermore these studies show that the CDX2 binding site appears to act as an enhancer, since its action is independent of its relative orientation and distance from the RNA polymerase II binding site. However, a very recent finding is that CDX2 may also interact directly with the TATA box. The mechanisms whereby tissue-specific transcriptional activation is achieved in the intestine will be discussed.
Genetic mapping of the doublefoot $(D b f)$ mutation

PASCALE-VALERIE GUILLOT, R. QUINNEY, P. H. GLENISTER, S. KERSCHER, Y. BOYD and M. F. LYON

Mammalian Genetics Unit, MRC, Harwell, Didcot, Oxfordshire OX11 ORD, UK

An $\mathrm{F}_{1}$ male offspring carrying a spontaneous mutation was found in a $\mathrm{C} 3 \mathrm{H} / \mathrm{HeH} \times 101 / \mathrm{H}$ cross mating. The mutation is characterized by extra toes on all four feet, hence the name doublefoot, $D b f$. Previous work has shown that the mutant allele is dominant and maps at a new locus on chromosome 1, between fuzzy $(f z)$ and leaden $(l n)$. Linkage tests with microsatellite markers located between $f z$ and $\ln$ (D1Mit22, D1Mit77, D1Mit24, D1Mit8) carried out on offspring from an interspecific backcross with Mus spretus showed that $D b f$ locus maps $7 \cdot 3 \pm 1 \cdot 8 \mathrm{cM}$ away from D1Mit22 and $5.9 \pm 1.6 \mathrm{cM}$ away from D1Mit8 and close to D1Mit24 and D1Mit77. It does not recombine with either of these markers, which in the Whitehead map are about $4 \mathrm{cM}$ apart. However, this difference is not an effect of $D b f$, as shown by results obtained in a control backcross. Among the genes mapped around the $D b f$ locus, $\operatorname{Pax} 3$ might be a candidate gene. However, the analysis of the segregation pattern of eight mice, known to be recombinant between D1Mit22 and D1Mit8 showed three recombinants between Pax3 and $D b f$, leading to Pax3 being ruled out as a candidate gene for the $D b f$ phenotype.

\section{Localization of the bronx waltzer (bv) deafness mutation and its effects on organ of Corti development in the ear}

T. BUSSOLI, A. KELLY and K. P. STEEL $M R C$ Institute of Hearing Research, University Park, Nottingham, NG7 2RD, UK

The bronx waltzer $(b v)$ mutation is an autosomal recessive mutation that is manifested as head tossing, circling and hearing impairment in the mouse. In the mouse inner ear, the inner hair cells (IHCs) and pillar cells of the organ of Corti and the maculae and cristae of the vestibular system are affected by this gene mutation. Abnormalities of IHCs have been reported as early as gestational day 17 (G17) and as few as $25 \%$ of IHCs have been reported in the adult organ of Corti. This study of the bronx waltzer organ of Corti confirms that IHCs begin to degenerate in the basal/middle coil of the cochlea between G17 and G18 and the same process occurs between birth and 4 days in the apical coil. We show that IHCs degenerate by a controlled mechanism of cell death. Secondary effects of IHC degeneration include a breakdown in the regular arrangement of pillar cells and outer hair cells (OHCs) and abnormal stereocilia arrangement 
on some misplaced OHCs. The $b v$ gene has not yet been identified. As a first step towards the positional cloning of this gene we established an intraspecific backcross between bronx waltzer and the mouse strain $101 / \mathrm{H}$. Using the polymerase chain reaction to amplify simple sequence length polymorphisms (SSLPs) from bronx waltzer and $101 / \mathrm{H}$ we were able to position the bronx waltzer gene to the middle region of mouse chromosome 5 . The $b v$ gene maps $1.33 \mathrm{cM}$ distal of the SSLP marker D5Mit $25,0.89 \mathrm{cM}$ proximal of D5Mit188 and co-segregates with D5Mit209 in 450 backcross progeny.

\section{Three X-linked mouse coat mutants}

E. GORMALLY, I. UWECHUE, H. BLAIR, T. HACKER and Y. BOYD

Mammalian Genetic Unit, MRC, Harwell, Didcot, Oxfordshire OX1 ORD, UK

During the evolution of mammals, genes mapping to the $\mathrm{X}$ chromosome have all remained on that chromosome even though their overall order has not been conserved. Consequently, X-linked mouse mutants have become very good tools and models for studying human X-linked genes and especially human genetic diseases. A number of mouse X-linked coat mutants have been reported and some of them have been proposed as possible models for human disorders. Data concerning three X-linked coat mutants will be reported. The mouse X-linked developmental mutant, tattered $(T d)$, has been shown to lie between DXMit55 and $X k h$ and is associated with hyperkeratinization. Two new X-linked coat mutants, faint line $(F n l)$ and stripey (Stpy), have also been studied. Genetic mapping of these mutants was undertaken to assess them as possible alleles of $T d$. Preliminary results from the mapping of $F n l$ places it between DXMit50 - DXMit81 and DXMit82 - DXMit105 - DXMit106 - DXMit159 - DXMit91 - DXMit193 and DXMit209 and therefore excludes $F n l$ as a possible allele of $T d$. Mapping of Stpy is in progress.

Genetic and physical mapping in the vicinity of the wi locus on mouse chromosome 4

A. J. W. PAIGE ${ }^{1}$, A. VARELA ${ }^{1}$, S. D. M. BROWN ${ }^{1}$, B. W. KIERNAN ${ }^{2}$, J. FLEMING ${ }^{2}$, M. ROGERS ${ }^{2}$, D. HUGHES ${ }^{2}$ and K. P. STEEL ${ }^{2}$

${ }^{1}$ MRC Mouse Genome Centre, Harwell, Oxfordshire, OX11 ORD; ${ }^{2} M R C$ Institute of Hearing Research, Nottingham NG7 2RD, UK

Whirler (wi) is a spontaneously arising mutation in mouse which results in deafness and vestibular dysfunction. Electrophysiological studies showed im- paired cochlear potentials which degenerate over time, and electron microscopy analysis of the inner ear revealed abnormal development of the inner hair cells. High resolution genetic maps in the vicinity of the wi locus have been constructed using large interspecific and intraspecific backcrosses segregating the wi mutation. The backcross progeny were genotyped with all the available markers on central mouse chromosome 4 and wi was found to be flanked by Ambp /D4Mit87 and Tnc, mapping $0 \cdot 13 \mathrm{cM}$ proximal and $0.39 \mathrm{cM}$ distal, respectively of $w i$. The gene $\operatorname{Orm} 1$ has been shown to be non-recombinant with wi in both crosses. In addition, the European Collaborative Interspecific Backcross (EUCIB) was used to generate a high-resolution genetic map of microsatellites across the wi region. We have successfully constructed a YAC clone contig across the wi region using a combination of TAIL PCR and vectorette PCR to recover YAC end clones. B1 IRS PCR has been used to recover new internal STSs to further define the wi critical region. In addition, a P1 clone contig is being constructed around Orm 1 and these clones are being used for a combination of exon trapping cDNA selection and sample sequencing to identify possible candidate genes for the wi mutation.

\section{Effects of leptin administration on lines of mice selected long-term for fatness}

LUTZ BÜNGER and WILLIAM G. HILL

Institute of Cell, Animal and Population Biology, University of Edinburgh, West Mains Road, Edinburgh EH9 3JT, UK

To assess the role of genetic changes in sensitivity to leptin hormone in contributing to responses to longterm selection for fatness, leptin was administered to a long-term fat selected (F) and a control line (C) of mice, which differ almost threefold in fat $\%$ at about 15 weeks of age. Treated (T) animals received twicedaily intraperitoneal injections of $5 \mathrm{mg} / \mathrm{kg}$ leptin from 91 to 105 days of age, untreated (U) animals received equivolume injections of PBS. Treated compared with untreated animals in both lines had significantly $(P<$ $0.05)$ lower body weight, food intake and fatness at the end of test (fat $\%$ : CT $3 \%$, CU $7.4 \%$, FT $14.9 \%$, FU $21.1 \%$ ). The differences in response between the lines $[(\mathrm{CT}-\mathrm{CU})-(\mathrm{FT}-\mathrm{FU})]$ were all non-significant $(P>0.05)$, however. There was a very wide range of fatness (estimated from dry matter content) among FT animals (3-29\%), much higher than in FU (15-31\%), CT (0.7-6.4\%) and CU (2-15\%). While sensitivity to leptin remains in the fat line, response appears to vary among animals at the dose level used. Preliminary results on the endogenous plasma leptin levels in untreated F-mice show very highly elevated levels compared with controls. 
The expression of the insulin-like growth factor family in human preimplantation embryos

\section{A. D. LIGHTEN, K. HARDY, R. M. L. WINSTON and G. E. MOORE \\ Institute of Obstetrics and Gynaecology, Royal Post- graduate Medical School, Hammersmith Hospital, Du Cane Road, London W12 ONN, UK}

Insulin, insulin-like growth factor-I (IGF-I) and insulin-like growth factor-II (IGF-II) are known to enhance growth in mouse preimplantation embryos in vitro. To study the role of the insulin-like growth factors in early human development, the timing of gene expression of insulin, IGF-I, IGF-II and their receptors was analysed. Reverse transcriptionpolymerase chain reaction (RT-PCR) was used to examine the presence of transcripts in preimplantation human embryos. Unfertilized human oocytes and spare human embryos following IVF were pooled in groups of five at the oocyte, 2-cell, 4-cell, 8-cell and blastocyst stages. Following reverse transcription, strategically designed nested primers were used for amplification from cDNA. Transcripts for the insulin receptor gene were present in unfertilized oocytes and in embryos from 8-cell stage onward, while transcripts for IGF1R and IGF2R were found at all stages of preimplantation development tested. In contrast, IGF2 was the only ligand expressed and transcripts were detected at all stages tested. This is consistent with expressed patterns seen in the mouse, where IGF-II is the only ligand in the family expressed and has been shown to have an autocrine effect on preimplantation development. We speculate that a similar relationship exists in the human and that preimplantation development may be regulated by IGFs from both embryonic (IGF-II) and maternal (insulin and IGF-I) sources.

\section{Detection of chromosome imbalance in human oocytes and first polar bodies using FISH}

J. COZZI ${ }^{1}$, C. CONN ${ }^{1}$, J. HARPER ${ }^{1,2}$ and J. D. A. DELHANTY ${ }^{1}$

${ }^{1}$ Human Genetics Group, The Galton Laboratory, University College London, London; ${ }^{2}$ Department of Obstetrics and Gynaecology, RPMS, Hammersmith Hospital, London, UK

Investigation of the chromosomal constitution of IVF unfertilized oocytes is a direct way to assess the contribution of female meiosis to chromosome abnormalities of the embryo. We have investigated with multi-colour FISH, aneuploidy for chromosomes 13, 18 and 21 in oocytes from routine IVF patients and chromosomal imbalance in oocytes from two patients referred for preimplantation diagnosis PGD because of a high risk of a down syndrome pregnancy. In the routine IVF patients, 2 out of 20 oocytes $(10 \%)$ were aneuploid for chromosome 21 with one having a missing chromatid 21 and one having an extra chromatid 21 . These oocytes probably resulted from precocious division of chromatids (PDC) at meiosis I. If fertilized they could have developed into trisomic embryos. Of the patients referred for PGD, case 1 was suspected maternal gonadal mosaicism for trisomy 21. Three of 4 oocytes analysed were hyperhaploid for chromosome 21 with two having an extra chromatid 21 and one having an extra whole chromosome 21. In one oocyte the presence of three signals for chromosome 21 in both oocyte and first PB confirmed the suspected gonadal mosaicism. Case 2 had a maternal reciprocal translocation $46, \mathrm{t}(6 ; 21)(\mathrm{q} 13 ; \mathrm{q} 22 \cdot 3)$. Two unfertilized oocytes were analysed, both of which had an unbalanced pattern of the translocation. Our results suggest that chromosome 21 may be predisposed to PDC which leads to oocytes with extra single chromatids and eventually to trisomic embryos. Patients undergoing PGD were shown to produce a high rate of unbalanced gametes, explaining the multiple chromosomally abnormal pregnancies they experienced.

\section{Accelerated growth and testicular differentiation: which comes first? \\ U. MITTWOCH \\ Department of Anatomy, Queen Mary and Westfield College, Mile End Road, London E1 4NS, UK}

Sex determination in mammals is thought to depend on a switch, committing the sexually undifferentiated gonad to enter the testicular or ovarian pathway, while testicular differentiation is envisaged as a cascade following the SRY switch. However, the bifurcation is asymmetrical, since the testis differentiates earlier than the ovary. It also grows faster. The question of whether accelerated growth precedes or follows testicular differentiation will be examined on the basis of growth data in the rat. In rat embryos, the beginning of Sertoli cell formation is seen on day 13, when XY gonads are about $35 \%$ larger than XX gonads. Accelerated growth of the testis continues until day 17 , when its volume has increased 200 -fold, compared with a 90 -fold increase of the future ovary, and the somatic sex of male embryos is largely established. The growth of both testis and ovary then drops, and ovarian differentiation begins. Since testicular and ovarian differentiation are associated with fast and slow growth respectively, growth retardation involving the XY gonad is liable to result in ovarian differentiation and sex reversal, even after testicular differentiation has begun. It is concluded that the question of whether fast growth precedes testicular differentiation, or vice versa, is irrelevant. 
Analysis of homologous chromosome pairing in mouse oocytes using a novel microchromosome

\section{CHARLES TEASE and GRAHAM FISHER \\ MRC Mammalian Genetics Unit, Harwell, Didcot, Oxforshire OX11 ORD, UK}

The morphology of nuclei in the early stages of meiosis has hampered analysis of the initial events of homologous chromosome pairing. One means to overcome this technical limitation is to exploit chromosome rearrangements. Recently, a microchromosome was found in the mouse that is composed principally of telomeric and satellite DNAs. This novel chromosome therefore provides a means of exploring the roles of these repeat sequence elements in the initiation of homologous chromosome pairing. Examination of early pachytene oocytes from fetal mice carrying two copies of the microchromosome showed that (1) in approximately $50 \%$ of oocytes the two microchromosomes lay in adjacent positions, and (2) in about $10 \%$ they had initiated synapsis. Thus although the microchromosomes are composed predominantly of common repeat sequence elements, they retain sufficient information to pair in a significant proportion of cells. Intriguingly, the behaviour of the microchromosomes is at variance with expectations from another study using FISH with repeat sequence DNA probes for the telomeres and pericentric satellites. The FISH analysis indicated that centromeric and telomeric chromosome ends did not play a significant role in the process of homology recognition. Possible explanations for the discrepant conclusions of these two studies will be considered.

Identification of a new imprinting region on distal mouse chromosome 2

CHRISTINE M. WILLIAMSON ${ }^{1}$, NOBUAKI $\mathrm{KIKYO}^{2}$, COLIN V. BEECHEY ${ }^{1}$, SIMON T. BALL $^{1}$, ROSALIND M. JOHN ${ }^{2}$, SHEILA C. BARTON $^{2}$, FUMITOSHI ISHINO ${ }^{3}$, CHARLES TEASE $^{1}$, ELIZABETH R. MARTIN ${ }^{1}$, BRUCE M. CATTANACH $^{1}$, M. AZIM SURANI ${ }^{2}$ and JOSEPHINE PETERS ${ }^{1}$

${ }^{1}$ Mammalian Genetics Unit, MRC, Harwell, Didcot, Oxfordshire, OX11 ORD, UK; ${ }^{2}$ Wellcome/CRC Institute of Cancer and Developmental Biology, and Physiological Laboratory, University of Cambridge, Tennis Court Road, Cambridge CB2 1QR, UK; ${ }^{3}$ Gene Research Center, Tokyo Institute of Technology, 4259 Nagatsuta-cho, Midoriku, Yokohama 226, Japan

Distal chromosome (Chr) 2 is subject to parental imprinting, as mice carrying maternal duplication/ paternal deficiency for distal Chr 2 or the reciprocal genotype exhibit phenotypic abnormalities. A new imprinted gene, neuronatin (Nnat), has been found on distal Chr 2 using an mRNA differential display method. Nnat was shown to be paternally expressed in the central and peripheral nervous system and appeared to be a good candidate for the hypoactive phenotype associated with maternal duplication of the region. We looked at the expression of Nnat in duplication/deficient mice for distal Chr 2. Results showed that Nnat was not expressed in the brain of neonates carrying maternal duplication of the region distal to the $\mathrm{T}(2 ; 8) 26 \mathrm{H}$ breakpoint in band $\mathrm{H} 1$ but was expressed in the brain of neonates carrying maternal duplication of the region distal to the $\mathrm{T}(2 ; 4) 1 \mathrm{Sn}$ breakpoint, also in band $2 \mathrm{H} 1$. These results place Nnat between the translocation breakpoints of $\mathrm{T} 26 \mathrm{H}$ and $\mathrm{T} 1 \mathrm{Sn}$ in band $2 \mathrm{H} 1$ and this mapping was verified by FISH and linkage analysis. Surprisingly, Nnat lies proximal to the reported minimal imprinting region located between $2 \mathrm{H} 3$ and $2 \mathrm{H} 4$, thus excluding Nnat as a candidate for the hypoactive phenotype and suggesting that there may be two distinct imprinting regions on distal Chr 2.

\section{Expression of PACAP receptor mRNA during early development of the mouse nervous system}

\section{W. J. SHEWARD, E. M. LUTZ and A. J. HARMAR MRC Brain Metabolism Unit, Royal Edinburgh Hos- pital, Morningside Park, Edinburgh EH10 5HF, UK}

Several lines of evidence suggest possible roles for vasoactive intestinal peptide (VIP), pituitary adenylate cyclase activating peptide (PACAP) or a related peptide during early development. We have examined the expression of mRNA for the receptors for VIP and PACAP in the mouse from day 9.5 of embryonic development onwards by reverse transcriptase PCR and by in situ hybridization. VIP1, VIP2 and PACAP receptor mRNAs were detected by PCR in the earliest developmental stage examined, and thereafter the levels of mRNA for all receptors increased during development. Whole-mount in situ hybridization first showed the expression of the PACAP receptor in the neural tube of 9.5-day-old embryos; in later embryos heaviest expression of the PACAP receptor was found in the developing rhombencephalon (days 10.5 and 11.5). These findings were confirmed and extended using autoradiographic in situ hybridization which showed also that PACAP receptor mRNA was expressed in the developing sympathetic chain and in the trigeminal ganglia. Although VIP2 receptor mRNA was detected by reverse transcriptase PCR from day 9.5 of embryonic development, the level of expression was too low to be visible by in situ hybridization. These results are consistent with a role for PACAP or a related peptide in the early development of the central nervous system. 
Molecular analysis of Loop-tail, a mouse model of craniorachischisis

DIANNE GERRELLI, NICHOLAS GREENE and ANDREW J. COPP

Neural Development Unit, Division of Cell and Molecular Biology, Institute of Child Health, 30 Guilford Street, London WC1N 1EH, UK

Loop-tail $(L p)$ is a semidominant mouse mutation closely resembling the human malformation craniorachischisis, a very severe form of neural tube defect. The defect that occurs in $L p / L p$ results from a failure in neural tube closure at the cervical-hindbrain boundary at the 6-8 somite stage. Demonstrating which molecules are defective in this mutant may aid our understanding of the cause of severe neural tube defects. Work in our laboratory has demonstrated that the notochord is wider, and the floor plate is enlarged, in $L p / L p$ embryos before the time of neural tube closure. To investigate the molecular mechanism underlying this abnormality we have compared patterns of gene expression between $L p / L p, L p /+$ and $+/+$ embryos before, during and following the time of normal neural tube closure. The results suggest that midline signalling by the notochord is disturbed in $L p / L p$, leading to an alteration in dorso-ventral patterning, somite patterning, an enlarged floorplate and consequent failure of neural tube closure.

The regulation of myogenin and $M y f 5$ expression in the developing mouse embryo

DENNIS SUMMERBELL, PETER ASHBY, DAVID COX, CHANDRIKA HALAI, SIU-POK YEE and PETER W. J. RIGBY

Division of Eukaryotic Molecular Genetics, MRC, National Institute for Medical Research, The Ridgeway, Mill Hill, London NW7 1AA, UK

The myogenic regulatory factors (MRFs) are a family of four basic-helix-loop-helix (bHLH) transcription factors each of which, when ectopically expressed in a variety of cultured cell types, can trigger the entire myogenic programme. During development the four genes are expressed in characteristic patterns and targeted mutagenesis studies show that each has a distinct role in myogenesis. We have previously shown that $133 \mathrm{bp}$ of myogenin $5^{\prime}$ flanking DNA recapitulates in transgenic mice the full temporal and spatial expression pattern in skeletal muscle precursors. This region contains two important motifs, which are consensus binding sites for bHLH proteins and MEF-2 proteins. Mutation of each site produces separate distinct phenotypes. The protein that binds to the bHLH site is almost certainly $M y f 5$, the first MRF to be expressed during embryogenesis. In marked contrast to myogenin, Myf5 has no discrete regulatory region. The control sequences are spread over at least $13 \mathrm{~kb}$ of DNA, including the adjacent gene MRF4. Further, there are distinct control regions for various muscle precursors, e.g. the epaxial domain, the hypaxial domain, the face and the limbs. That Myf 5 has separate control regions for different muscle blocks, presumably controlled by different inductive signals, seems highly appropriate for a determination gene.

Analysis of gene expression during preimplantation embryonic development in humans: the construction and use of PCR-generated cDNA libraries

JAMES ADJAYE ${ }^{1}$, ROBERT DANIELS ${ }^{1}$, VIRGINIA BOLTON ${ }^{2}$ and MARILYN MONK ${ }^{1}$

${ }^{1}$ Molecular Embryology Unit, Institute of Child Health, 30 Guilford Street, London WC1N 1EH; ${ }^{2}$ Department of Obstetrics and Gynaecology, King's College School of Medicine and Dentistry, Denmark Hill, London SE5 8RX, UK

Human preimplantation embryos for use in research are rare and only obtainable by very few laboratories licensed to work on this precious material. We propose to make the molecular analysis of early human development accessible to all by constructing stagespecific (oocytes, 2-cell, 4-cell, 8-cell and blastocyst stage embryos) and tissue-specific (trophectoderm and inner cell mass from blastocysts) cDNA libraries. We present data on the construction and analysis of a lambda-ZAPII cDNA library derived from a single 4cell human embryo. These libraries will provide a readily available resource for the study of the timing and tissue-specificity of gene activity in early development, which will obviate the need for fresh human embryonic material. Such a resource may be expected to increase our basic understanding of development at the molecular level and, more specifically, to give information beneficial to genetic diagnosis and the onset of specific genetic diseases.

The role of the extracellular matrix in neural crest cell migration in the Splotch ${ }^{2 \mathrm{H}}$ mouse

\section{DEBORAH J. HENDERSON and ANDREW J. COPP}

Neural Development Unit, Division of Cell and Molecular Biology, Institute of Child Health, 30 Guilford Street, London WC1N 1EH, UK

The Splotch phenotype arises as a consequence of mutations in the Pax3 gene, a developmentally regulated transcription factor. The defects found in the $S p^{2 H}$ mouse mirror the expression pattern of $\operatorname{Pax3}$, and include the neural tube defects spina bifida and exencephaly, neural crest cells (NCC) abnormalities 
such as reduced or absent dorsal root ganglia and persistent truncus arteriosus, and absence of limb musculature. We have been concentrating on the extracellular matrix as a potential cause of failure of neural crest cell migration in this mutant. Versican is a chondroitin sulphate-bearing proteoglycan which is expressed in tissues through which NCC migrate, although it is inhibitory to this migration. We have shown that versican is greatly over-expressed in $S p^{2 H}$ embryos between 9.5 and 11.5 days of gestation when the NCC are migrating. In addition the expression of versican is reciprocal to that of Pax3 in both the wildtype and mutant embryos, such that where $\operatorname{Pax} 3$ is absent in the mutant there is ectopic expression of versican. This suggests that versican may be regulated by $P a x 3$ and that the over-expression of versican, as a consequence of the absence of functional $\operatorname{Pax} 3$ in $S p^{2 H}$ embryos, results in inhibition of neural crest cell migration in this mutant.

\section{Role of Hoxb-1 during hindbrain specification}

M. STUDER ${ }^{1}$, A. LUMSDEN ${ }^{2}$, L. ARIZAMCNAUGHTON $^{1}$, A. BRADLEY ${ }^{3}$ and R. KRUMLAUF $^{1}$

${ }^{1}$ Laboratory of Developmental Neurobiology, NIMR, Mill Hill, London, UK; ${ }^{2}$ Department of Developmental Neurobiology, Guy's Hospital, London, UK; ${ }^{3}$ Howard Hughes Medical Institute, Baylor College of Medicine, Houston, Texas, USA

During development, the hindbrain is gradually subdivided into rhombomeres (r), which is critical for the organization and innervation of the cranial region. Functional analysis in mice has proposed vertebrate Hox genes as main players during the A-P specification of the embryonic body plan, but their role in the hindbrain is still unclear. Hoxb- 1 is the earliest member of the Hox-b complex to be expressed in the embryo and is strongly upregulated in $\mathrm{r} 4$, suggestive of a role in the specification of $\mathrm{r} 4$ identity. We now show that mice lacking Hoxb-1 die just after birth and present specific neuronal defects in the hindbrain region. Axon-tracing experiments and extensive analysis with molecular markers are indicative of a perturbation in r4 identity. Moreover, a specific population of neurons of the facial (VII) cranial nerve located normally along the midline of $\mathrm{r} 4$ and $\mathrm{r} 5$ fail to migrate and no contralateral vestibulo-acoustic efferent axons cross the floor plate of r4. Later in development the entire facial motor nucleus is lost coincident with an increase in apoptosis. The data demonstrate that as part of its role in maintaining segmental identity, Hoxb-1 is involved in controlling the guidance and migration of motor neurons in the hindbrain. 